Research Article

\title{
Volume Changes and Mechanical Properties of Expansive Mudstone below Canals under Wet-Dry/ Wet-Dry-Freeze-Thaw Cycles
}

\author{
Rui Zhu $\mathbb{D}^{1,2,3}{ }^{1,3 i n g-h a o ~ H u a n g, ~}{ }^{3}$ Zhu Song, ${ }^{1}$ and Feng Zhou $\mathbb{D}^{1,2}$ \\ ${ }^{1}$ School of Transportation Engineering, Nanjing Tech University, Nanjing 211816, China \\ ${ }^{2}$ Key Laboratory of Ministry of Education for Geomechanics and Embankment Engineering, Hohai University, \\ Nanjing 210098, China \\ ${ }^{3}$ Geotechnical Engineering Department, Nanjing Hydraulic Research Institute, Nanjing 210024, China
}

Correspondence should be addressed to Feng Zhou; zhoufeng@njtech.edu.cn

Received 18 April 2021; Accepted 26 May 2021; Published 7 June 2021

Academic Editor: Rihong Cao

Copyright (C) 2021 Rui Zhu et al. This is an open access article distributed under the Creative Commons Attribution License, which permits unrestricted use, distribution, and reproduction in any medium, provided the original work is properly cited.

\begin{abstract}
The complex environment in northern China is the main reason for degradation of expansive mudstone below the canals, which resulted in instability and damage of canal slopes. In this study, a serial of laboratory tests was conducted to explore the volume changes and mechanical behaviors of expansive mudstone below the canals in Xinjiang. The experimental program includes wet-dry (WD) and wetdry-freeze-thaw (WDFT) tests, volume measurement, and unconfined compression tests. The test results show that during the WD cycles, the volume changes of expansive mudstones with a higher dry range would be more significant. The freeze-thaw process in the WDFT cycles resulted in a decrease of volume change ranges when the expansive mudstones had a relatively smaller dry range and a slight increase of volume change ranges when the expansive mudstones had a relatively larger dry range. In the meantime, the stressstrain relationships of expansive mudstones with different dry ranges all presented strain softening under the cycles of WD or WDFT. The first cycle resulted in a significant decrease of failure strength. After seven WD/WDFT cycles, the failure strength of expansive mudstones with different dry ranges decreased by $37.2 \%$ 59.1\%. In addition, the freeze-thaw process in the WDFT cycles promoted the softening of the stress-strain relationships and aggravated the failure strength attenuation of expansive mudstones. Through this study, we expect to provide a preliminary basis for the construction and maintenance of expansive mudstone canals in Xinjiang.
\end{abstract}

\section{Introduction}

Long-distance water transfer projects are a major strategy to realize the optimal allocation of water resources, and water transfer canals are the main methods used in water transfer projects. China has approximately 4.5 million $\mathrm{km}$ of various water transfer canals, but the utilization coefficient of canal water is relatively low [1]. Canals in northern China are particularly susceptible to damage by the harsh natural environment, resulting in hidden dangers to the stable operation of the canals [2,3], as shown in Figure 1.

Wet-dry cycles and freeze-thaw cycles are two typical boundaries simplified from natural environment of on-site canals. On this basis, a large number of experimental studies have been conducted on volume changes and mechanical degradation of foundation soils below the canals under wetdry and freeze-thaw cycles [4-8]. Lu et al. $[9,10]$ investigated the freeze-thaw performance of the expansive soil taken from South-to-North Water Transfer Project by UCS and SEM tests. It is found that the internal porosity of expansive soil increased and became more uniform after several freezethaw cycles, which is more significant among the soils with a higher moisture content. Wang et al. [11, 12] studied the mechanical characteristics of saline soil under different moisture contents, salt contents, and freeze-thaw cycles, which provide a basic reference for canal construction in Jilin. Li et al. [13-15] investigated the frost damage mechanism of foundation soil in cold regions, which is helpful to theoretical and numerical studies. Recently, some innovative instruments, such as NMR, CT, and TDR, have been used in 

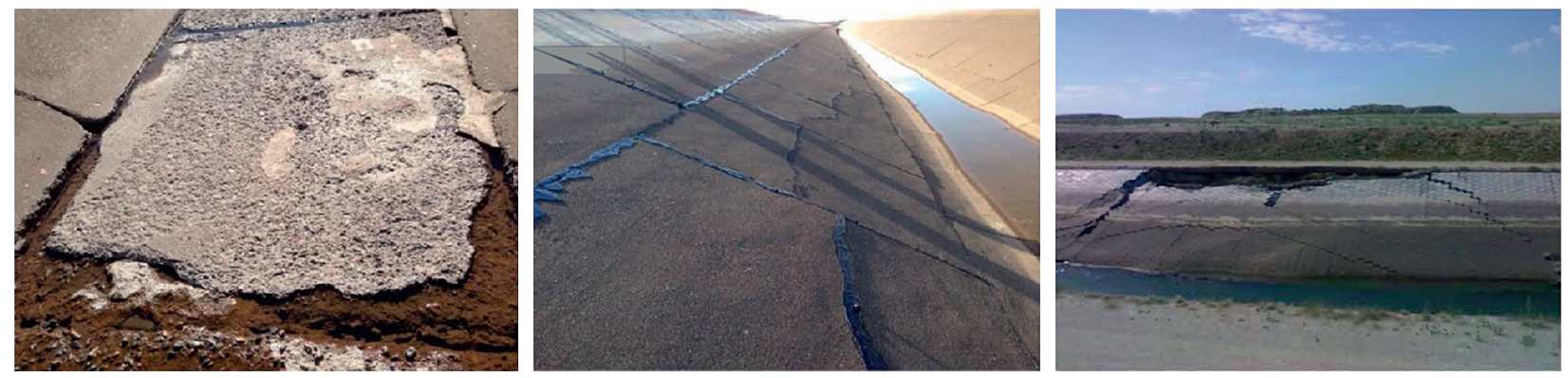

Figure 1: Typical damages of canals in northern China $[2,3]$.

frozen soil studies [16-18], which greatly perfect the quantitative analysis of unfrozen water content and microstructure development [19-22]. Additionally, Zhu et al. [23] investigated the relationships between surface cracks and mechanical properties of expansive soil and then analyzed the damage characteristics of canal slopes under wetdry cycles by centrifugal model tests $[24,25]$.

The above studies play an important role in the mechanism investigation of foundation soil below the canals under cyclic actions of wet-dry or freeze-thaw [26-28]. However, studies related to a typical canal in Xinjiang showed that the expansive mudstone below the canals experienced a wet-dry-freeze-thaw process throughout the year [29-31], as shown in Figure 2. The volume changes and mechanical properties of expansive mudstone below the canals in Xinjiang are very scarce in the literature. Consequently, current studies cannot provide a well reference for these canals.

Accordingly, the objective of this study is to investigate the volume changes and mechanical properties of expansive mudstone below the canals in Xinjiang. Specially, a series of wet-dry (WD)/wet-dry-freeze-thaw (WDFT) tests and unconfined compressive strength tests were performed, and then, performances of deformation and strength upon the dry ranges and cycle numbers of WD/WDFT were analyzed. Through this study, we expect to provide a preliminary basis for the construction and maintenance of expansive mudstone canals in Xinjiang.

\section{Materials}

The samples tested in this study were prepared with a natural expansive mudstone material, which was taken from the field of a typical canal in Xinjiang [29, 30], as shown in Figure 3. Some fundamental properties of the mudstone were measured following procedures described in the specifications [32-34], as given in Table 1. In the meantime, the grain size distribution was analyzed using sieving analysis [35] and the hydrometer method in the laboratory [36], as shown in Figure 4. It is found that the percent passing \#200 sieve is as high as approximately $69 \%$.

\section{Experimental Program}

3.1. Sample Preparation. Before preparation, the original soil material was air-dried for about two weeks. Then, it was grinded and sieved through a $2.0 \mathrm{~mm}$ sieve. The sieved soil was stored in buckets. According to the test scheme, the soil was added with water in measured quantities and mixed completely until initial moisture content reached $18.4 \%$, which is the optimum moisture content. The mixed soil was sealed in plastic bags for approximately 24 hours for a homogeneous soil moisture distribution. Next, the soil was compacted using a stratified sample preparation device and prepared samples with $39.1 \mathrm{~mm}$ in diameter and $80 \mathrm{~mm}$ in height, as shown in Figure 5. Six samples for each condition were prepared. Among them, three samples were for the measurement of volume changes and three samples were for unconfined compression tests, which significantly reduced the disturbance of the samples for mechanical tests $[9,10,37]$. All the samples were compacted to the same dry density of $1.60 \mathrm{mg} / \mathrm{m}^{3}$, which is on the basis of the field test results [23-25]. After compaction, the samples were extracted from the mold for the WD and WDFT tests.

3.2. WD and WDFT Tests. After sample preparation, all the samples were divided into two series: half of the samples experienced the repeated WD processes while the other part underwent the WDFT cycles. Both of the WD cycles and the WDFT cycles were $0,1,3$, and 7 cycles, which was decided because the soil properties tended towards stability after 3 WD cycles $[38,39]$. The wet, dry, freeze, and thaw processes were as follows:

\section{(1) Wet process}

The wet process corresponds to the operation period of the typical canal. During this period, the foundation soil below the canals was saturated due to the water infiltration. Consequently, the wet process of the samples was simulated by the vacuum saturation method.

(2) Dry process

The drying process corresponds to the nonoperation period of the typical canal. During this period, there is no water inside the canal, resulting in that the foundation soil below the canals was air-dried continuously. Thus, the samples were air-dried in the test room with a temperature of $20 \pm 1^{\circ} \mathrm{C}$ and a relative humidity of $55 \pm 5 \%$. The saturation variations of samples were monitored by weight, and the dry process would be terminated until it came to the target saturation $\left(S_{t}\right)$. 


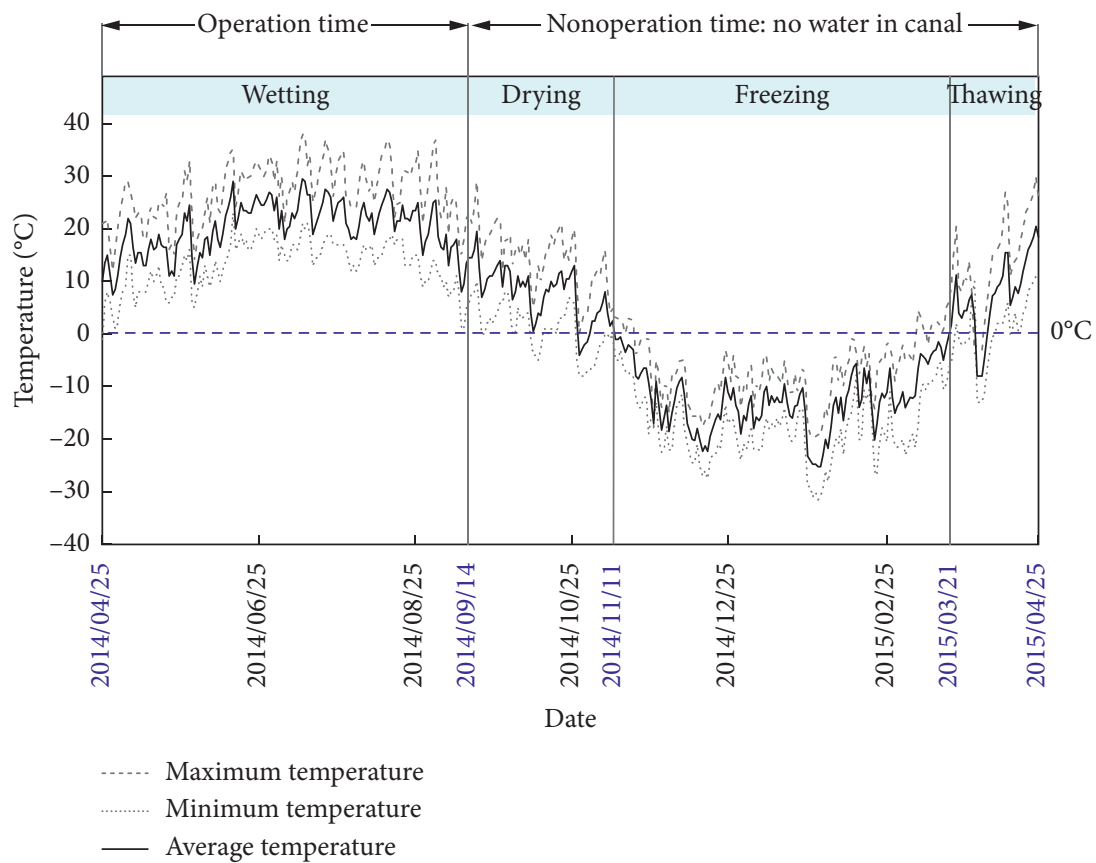

Figure 2: Temperature observed by a weather station along the canal in Xinjiang [29-31].

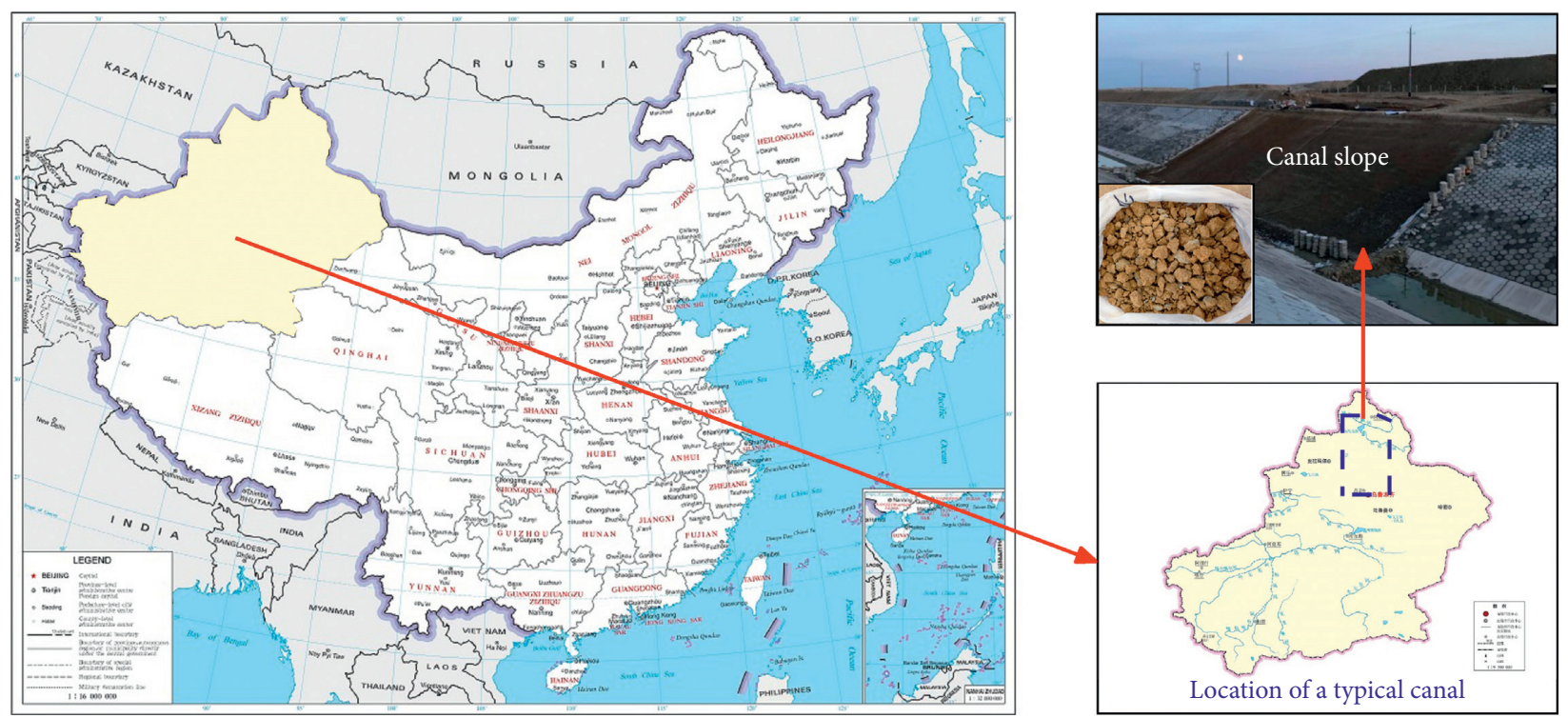

FIgURE 3: Location of the sampling area in Xinjiang, China.

(3) Freeze process

The freeze process corresponds to the time when the field temperature was lower than $0^{\circ} \mathrm{C}$. Therefore, the freeze process of the samples was simulated by the low-temperature environment test chamber in Nanjing Hydraulic Research Institute, as shown in Figure 6. The samples were wrapped with plastic film first, which was effective to avoid moisture loss in the freeze process and thaw process in the previous studies $[9,10]$. Then, the wrapped samples were exposed to three-dimensional closed environment with a consistent temperature of $-20^{\circ} \mathrm{C}$ for 12 hours.
(4) Thaw process

The thaw process corresponds to the time when the field temperature was higher than $0^{\circ} \mathrm{C}$. Thus, the thaw process of the samples was simulated by thawing at the room temperature of $20^{\circ} \mathrm{C}$ for 12 hours.

It is noted that the $-20^{\circ} \mathrm{C}$ in the freeze process and $20^{\circ} \mathrm{C}$ in the thaw process were derived from the average temperature in the field during winter and the average temperature in the field during summer, respectively. The time of the freeze process and thaw process was decided because 12 hours proved that it 
TABLE 1: Fundamental properties of mudstone material.

\begin{tabular}{lc}
\hline Properties & Value \\
\hline Specific gravity & 2.67 \\
Potential expansion & 71.0 \\
CHCS classification & $\mathrm{CH}$ \\
\hline Consistency limit & \\
Liquid limit & $52.6 \%$ \\
Plastic limit & $18.4 \%$ \\
Plasticity index & 34.2 \\
\hline Compaction study & \\
Optimum moisture content & $18.4 \%$ \\
Maximum dry density & $1.70 \mathrm{mg} / \mathrm{m}^{3}$ \\
\hline Mineral components & \\
Montmorillonite & $61.5 \%$ \\
Quartz & $31.9 \%$ \\
Feldspar & $6.1 \%$ \\
Calcite and albite & $0.5 \%$ \\
\hline
\end{tabular}

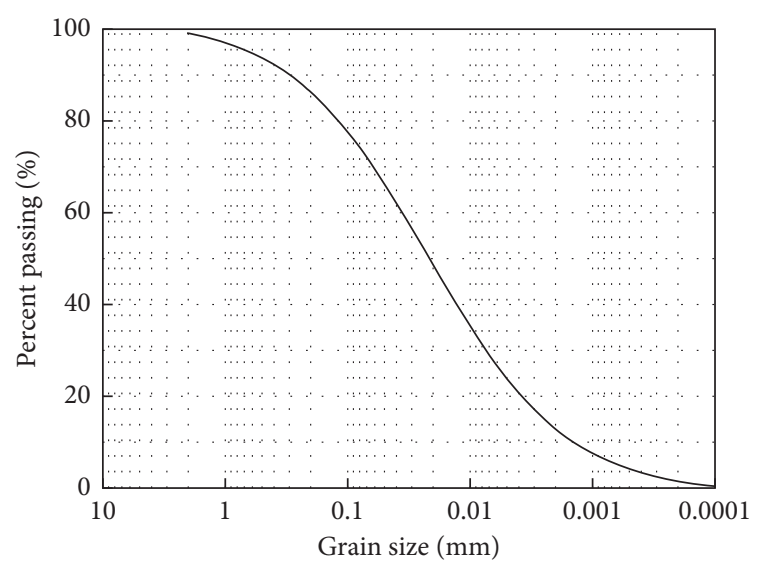

FIGURE 4: Grain size distribution.

is an adequate period after which the volume changes would become constant $[9,10]$. Additionally, the target saturation $\left(S_{t}\right)$ of samples was a variable in the dry process, resulting in that the sample saturation changed during the cyclic WD processes and WDFT processes, which are given in Table 2.

3.3. Measurement of Volume Changes and Unconfined Compression Strength Tests. In order to obtain the volumes changes of samples exposed to WD cycles and WDFT cycles, the diameter $(D)$ and height $(H)$ of samples were measured after each period of wet, dry, freeze, and thaw, since the sample deformation was basically homogeneous along the sample height. The measurement of sample dimensions using an electronic vernier caliper has a precision of $0.01 \mathrm{~mm}$, as shown in Figure 7. Additionally, the measured dimensions of samples were derived from the three values along cross-section and two values along the longitudinal section. Consequently, the average values of sample volume were calculated on the basis of the above measured values of diameter and height during the cyclic WD and WDFT processes.
The strength of samples was measured by an YSH-2 unconfined compression device in Nanjing Hydraulic Research Institute (Figure 8), which can obtain a great quantity of data to describe the mechanical behaviors of samples. The maximum load capacity and maximum strain rate of this device are $5 \mathrm{kN}$ and $1 \mathrm{~mm} / \mathrm{min}$, respectively. The samples were deformed under a compression load until the axial strain of samples reached $16 \%$. During this process, the strain rate was kept at $0.8 \mathrm{~mm} / \mathrm{min}$, which is equal to $1 \% /$ min. Consequently, the stress-strain response, resilient modulus, and failure strength of samples subjected to WD cycles and WDFT cycles can be obtained. Figure 9 shows the typical failure characteristics of samples with different dry ranges.

\section{Test Results}

4.1. Volume Changes. In order to characterize the volume changes of samples better, a dimensionless parameter, named volumetric strain $\left(\varepsilon_{\mathrm{v}}\right)$, was proposed to indicate relative volume change of samples during the cyclic WD and WDFT processes. The volumetric strain can be calculated as

$$
\begin{aligned}
\varepsilon_{V} & =\frac{\Delta V}{V_{0}}, \\
& =\frac{\left(V_{N}-V_{0}\right)}{V_{0}},
\end{aligned}
$$

where $V_{N}$ is the sample volume after $N$ cycles of WD or WDFT, and $V_{0}$ is the initial volume of samples after preparation. Consequently, the positive values of volumetric strain indicated volume expansion, while the negative ones represented volume contraction. Figure 10 shows the curves of volume changes variation with WD and WDFT cycles. Among the horizontal axis, 0 represents the initial state of samples, and 1, 2, 3, 4, 5, 6, and 7 refer to the completion of corresponding cycles of WD or WDFT.

As shown in Figure 10(a), the samples presented the typical characteristics of volume expansion in the wet process and volume contraction in the dry process, and the sample volumes changed alternately with the increasing WD cycles. Specifically, the volume variation trend of samples with dry range I during each WD cycle is relatively constant, especially after four WD cycles. Additionally, the volumetric strain of samples with dry range II during each WD cycle showed the similar variation characteristics to the ones with dry range I. However, the volumetric strain of samples with dry range II decreased gradually, resulting in a downward trend of sample volumes after each WD cycle. Different from the above samples with dry range I and dry range II, the volumetric strain of samples with dry range III during the wet process and dry process decreased significantly with the increasing WD cycles. Compared with the volumetric strain in the first WD cycle, the volumetric strain of samples with dry range III in the seventh cycle decreased by $65.8 \%$. In the meantime, it is also found from Figure 10(a) that the samples with dry range I showed the largest volumetric strain in a single WD cycle, indicating that the volume changes of 

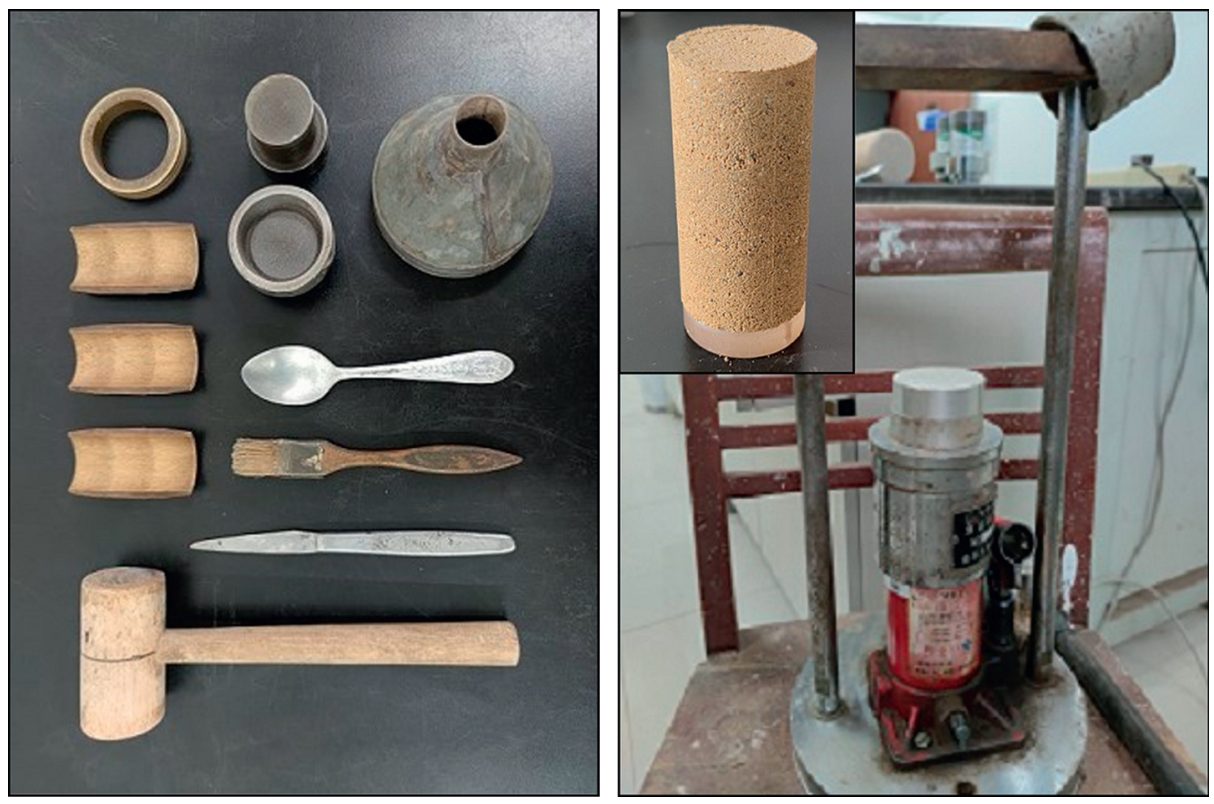

FIGURE 5: Stratified sample preparation device.

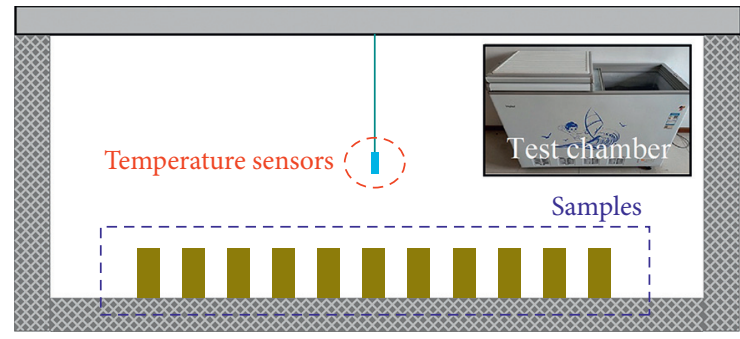

Figure 6: Low-temperature environment test chamber.

TABLE 2: Saturation variations of sample during the WD/WDFT processes.

\begin{tabular}{lcccc}
\hline \multirow{2}{*}{ Processes } & Wet & Dry & Freeze & Thaw \\
& $S_{i}$ & $S_{t}$ & $S_{t}$ & $S_{t}$ \\
\hline Sample & & $30 \%$ (dry range I) & $30 \%$ & $30 \%$ \\
\multirow{3}{*}{ Saturation } & $100 \%$ & $70 \%$ (dry range II) & $70 \%$ & $70 \%$ \\
& $90 \%$ (dry range III) & $90 \%$ & $90 \%$ \\
& In the dry process, different $S_{t}$ means different dry \\
Remarks & ranges. Among them, dry range I represents that $S_{t}$ \\
& is 30\%; dry range II represents that $S_{t}$ is $70 \%$; dry \\
& \multicolumn{3}{c}{ range III represents that $S_{t}$ is $90 \%}$. \\
\hline
\end{tabular}

samples with a larger dry range would be more significant, which is consistent with that reported in the literature [40].

Figure 10(b) shows the volume changes of samples exposed to WDFT cycles. It is found that the volumetric strain of samples with different dry ranges all present the variation characteristics of fluctuation during the cyclic WDFT processes. Among them, the volumetric strain of samples with dry range I after each WDFT cycle is relatively stable and basically remains constant after three WDFT cycles, while the volumetric strain of samples with dry range
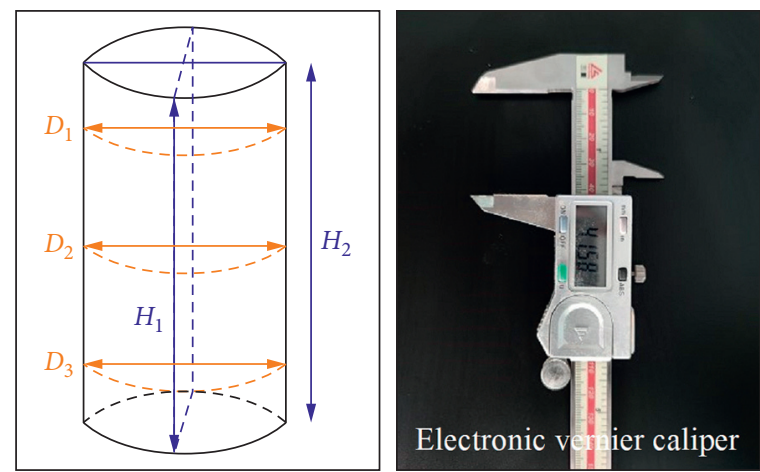

FIGURE 7: Schematic diagram of sample dimension measurement.

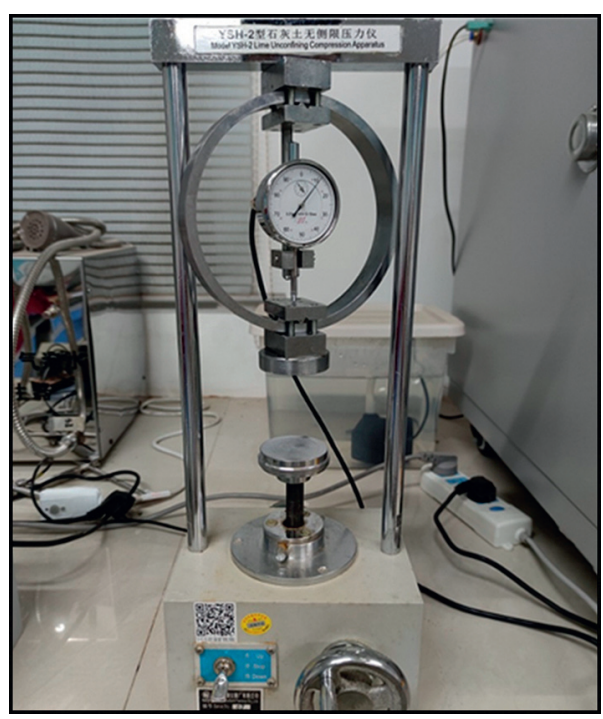

Figure 8: Device for unconfined compression. 


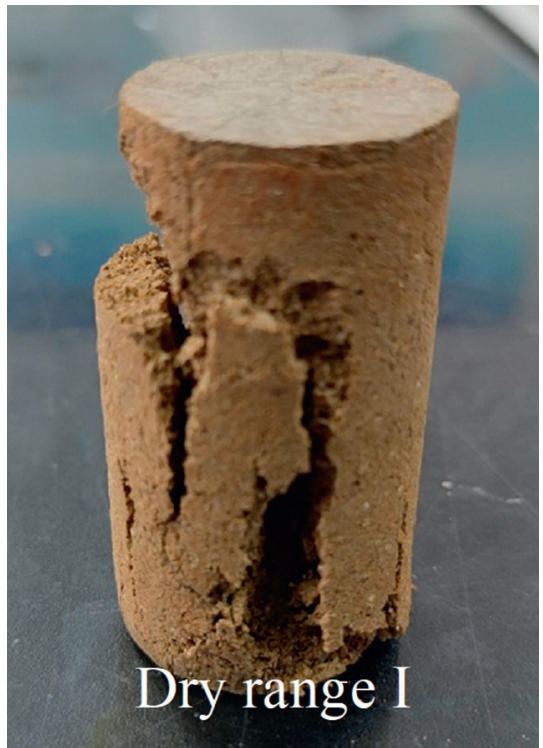

(a)

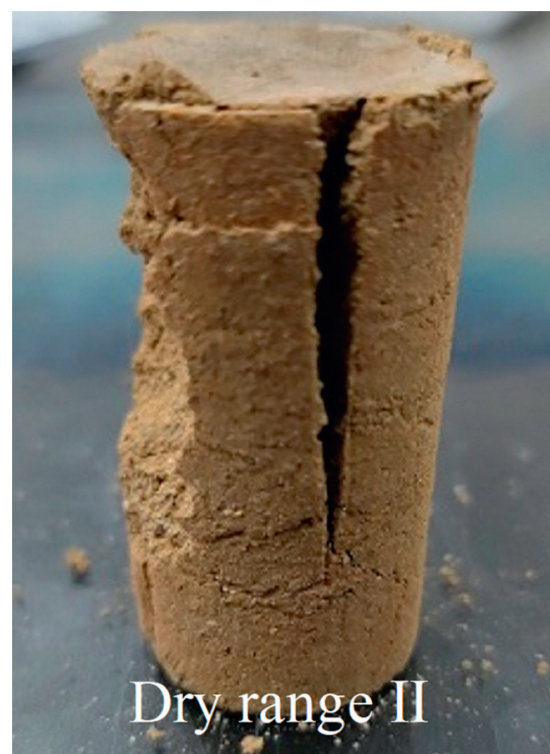

(b)

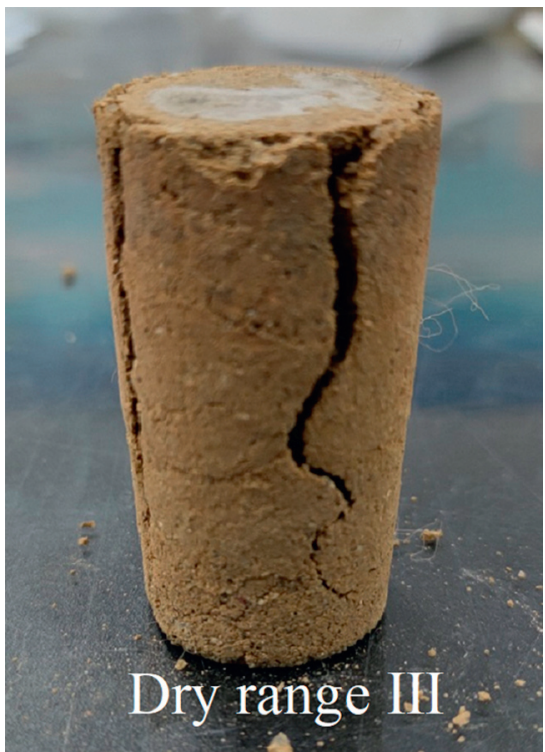

(c)

Figure 9: Typical failure characteristics of samples subjected to WDFT cycles.

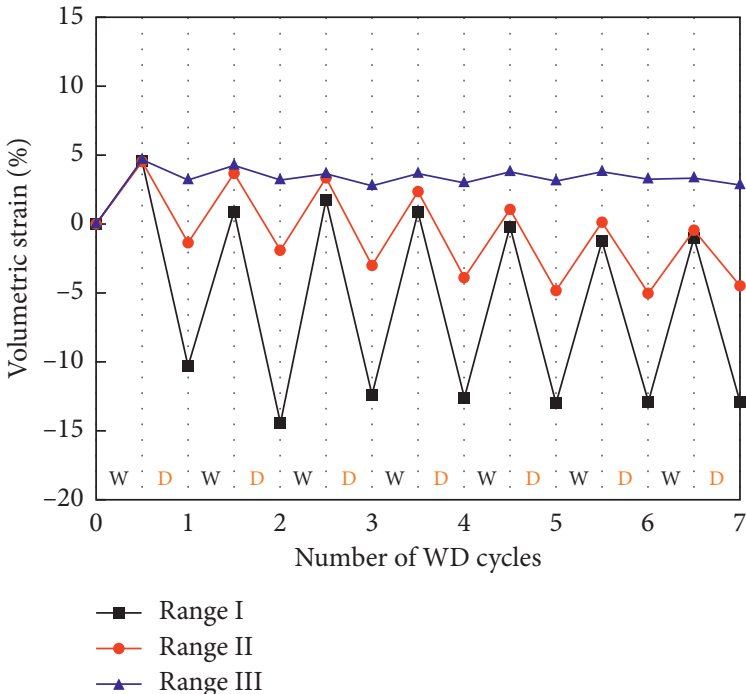

(a)

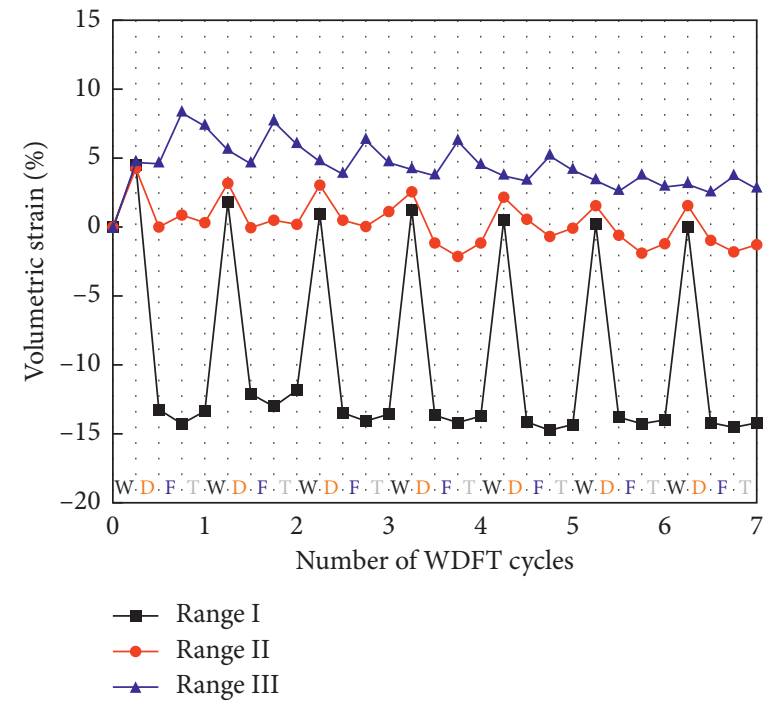

(b)

FIgURE 10: Curves of volume changes with cycles under different dry ranges. (a) Samples exposed to WD cycles. (b) Samples exposed to WDFT cycles.

II and dry range III after each WDFT cycle presented a downward trend with the increasing WDFT cycles. In addition, the samples with dry range I showed a significant wet-dry effect on the volume changes, which is reflected in a higher volume changes in the wet-dry process during the WDFT cycles than that in the freeze-thaw process during the WDFT cycles. Conversely, the samples with dry range III showed the freeze-thaw effect on the volume changes, which means that the volumes of samples with dry range III changed significantly in the freeze-thaw process during the WDFT cycles than that in the wet-dry process during the WDFT cycles. As previously mentioned, the volume changes of samples with dry range III presented a downward trend, indicating that the freeze-thaw effect weakened gradually with the increasing WDFT cycles, which is consistent with the volume change characteristics of expansive soils under freeze-thaw cycles reported in the literature [41]. The samples with dry range II, which is between dry range I and dry range III, presented a different volume change variation under WDFT cycles. At the beginning of WDFT cycles, the samples with dry range II had larger variation ranges of volumetric strain in the wet-dry process during the WDFT cycles, indicating a more significant wet-dry effect on the volume changes in the cyclic WDFT processes. With the 


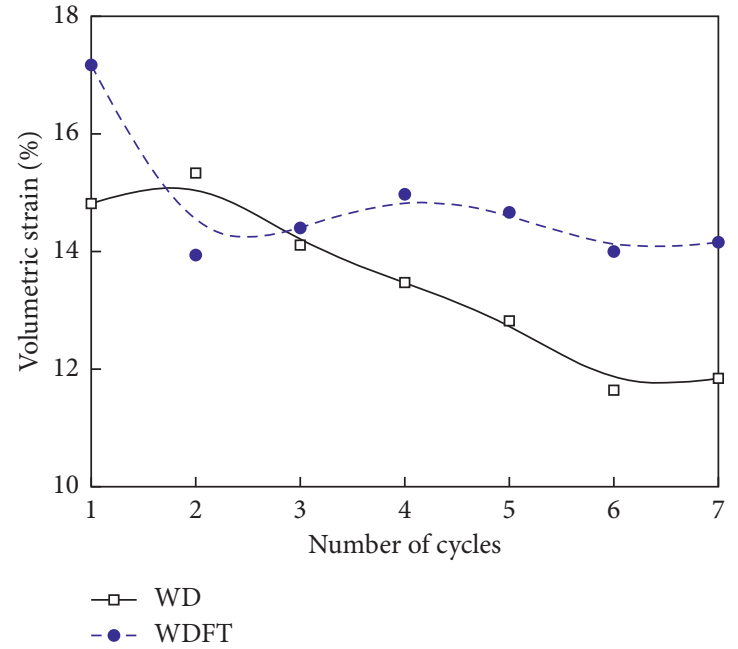

(a)

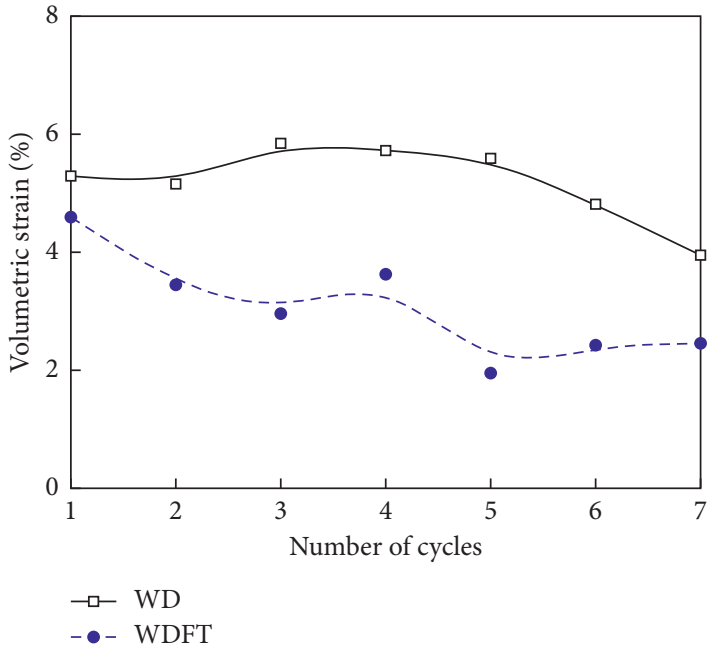

(b)

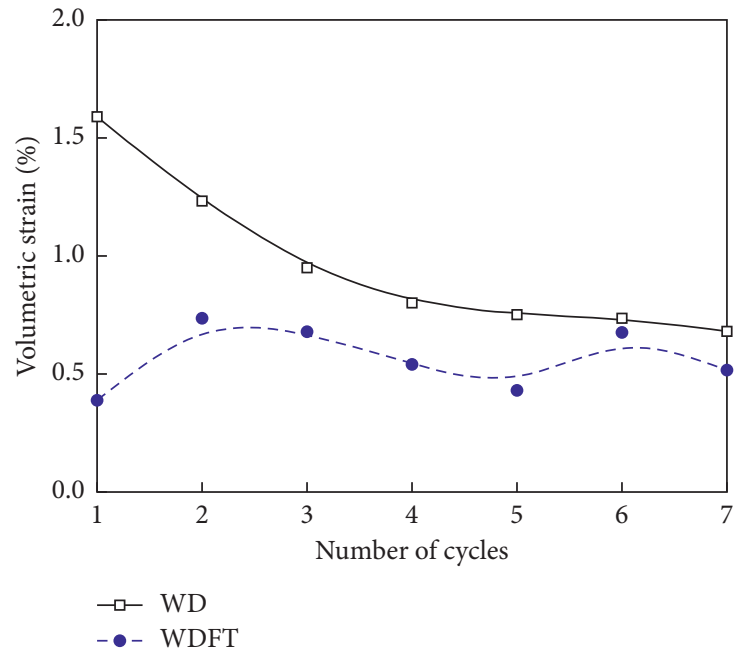

(c)

FIGURE 11: Comparison curves of volume changes under WD and WDFT cycles. (a) Dry range I. (b) Dry range II. (c) Dry range III.

increase of WDFT cycles, the volumes of samples with dry range II changed more significantly in the freeze-thaw process during the WDFT cycles, indicating that the freezethaw effect gradually dominated the volume changes of samples during the cyclic WDFT processes. This phenomenon indicated that there may be a coupled effect between the wet-dry process and freeze-thaw process when the samples with a medium dry range were exposed to WDFT cycles, which was never reported in the previous studies.

It was noted that the volumetric strain of samples with dry range I and dry range II showed a downward trend in the freeze process and a rising trend in the thaw process. The main reason for this phenomenon is as follows: the saturation of samples with dry range I and dry range II is relatively low after the dry process. During the freezing process, a large number of air in the pores and the water in the sample turned into ice, leading to an increase of sample volumes. In the meantime, the expansive mudstone particles lost water and shrink, and the shrinkage would be large due to the sufficient shrinkage space, which offset the frost heave. Consequently, the macrovolume of samples decreased in the freeze process. In the thaw process, the ice in the pores thawed into water. Concurrently, the expansive mudstone particles expanded with water, and the expansion was greater than the shrinkage. Therefore, the macrovolume of samples would increase in the thaw process $[9,10]$.

For the purpose of quantitatively analyzing the difference of volume changes with WD cycles and WDFT cycles, the relationships between the difference of the maximum volumetric strain and the minimum volumetric strain during each cycle and the number of cycles are plotted, as shown in Figure 11. It is found that with the increasing cycles, the volume changes of samples with dry range I under WD cycles were more significant than that under WDFT cycles, while the samples with dry range II and dry range III showed the opposite phenomenon. It indicated that the freeze-thaw process in the WDFT cycles resulted in a decrease of volume change ranges when the samples had a 

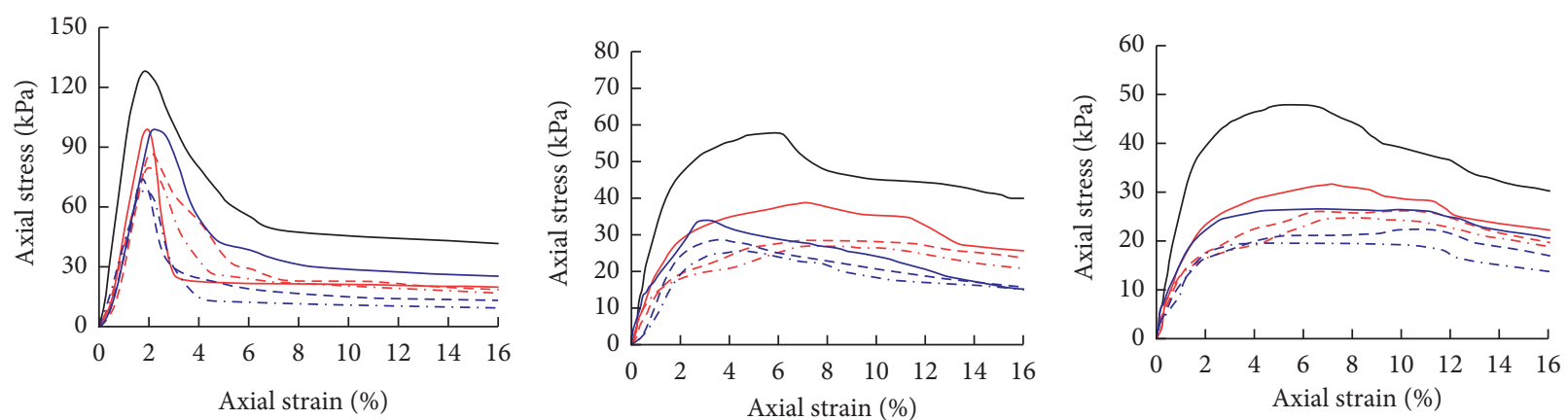

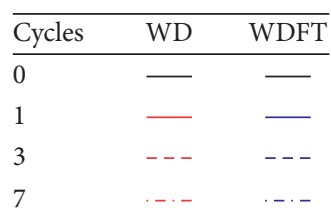

(a)

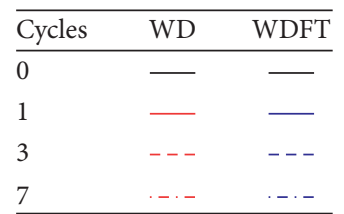

(b)

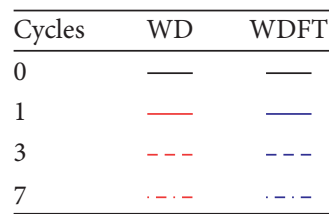

(c)

Figure 12: Curves of stress-strain relationships. (a) Dry range I. (b) Dry range II. (c) Dry range III.

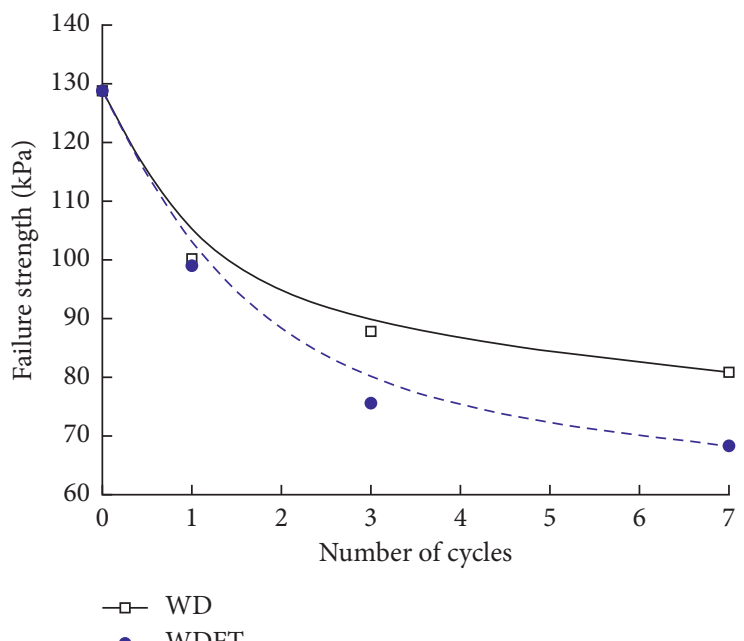

(a)

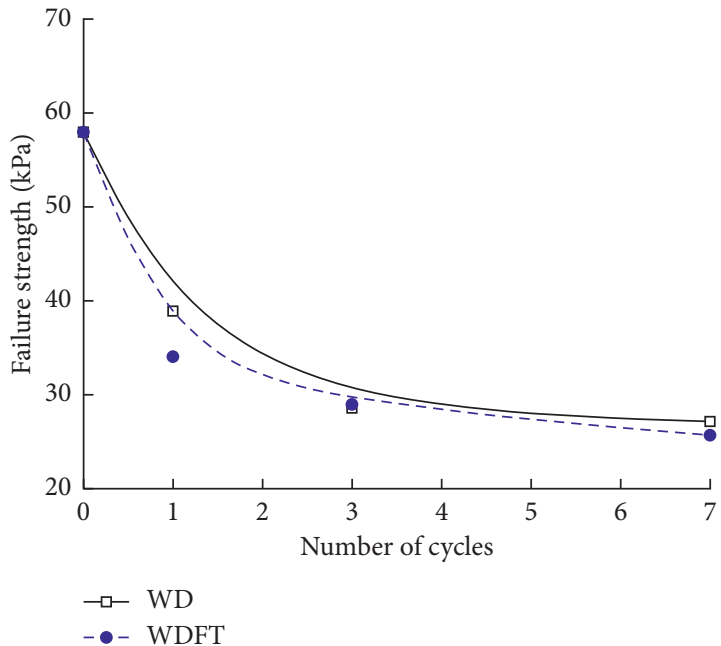

(b)

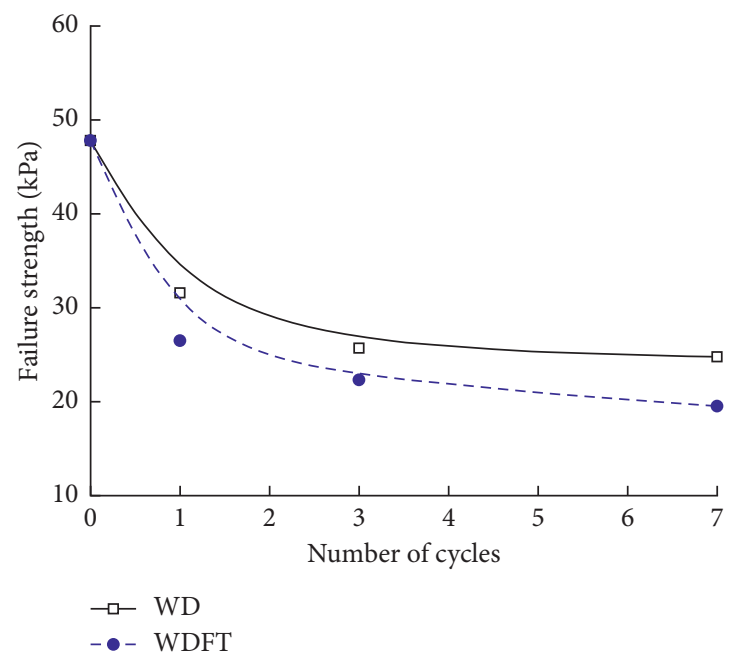

(c)

Figure 13: Curves of failure strength variation with cycles. (a) Dry range I. (b) Dry range II. (c) Dry range III. 
relatively smaller dry range and a slight increase of volume change ranges when the samples had a relatively larger dry range. This is also consistent with the volume change characteristics of shallow foundation soils below the canal with a large dry range in Xinjiang [29, 30].

\subsection{Mechanical Behaviors}

4.2.1. Stress-Strain Behaviors. Unconfined strength tests were conducted on samples exposed to WD cycles and WDFT cycles. To compare the characteristics of stress-strain behaviors, the stress-strain relationships of samples subjected to $0,1,3$, and 7 cycles of WD and WDFT are plotted as shown in Figure 12. It is found that under WD and WDFT cycles, the stress-strain relationships of samples with different dry ranges all presented strain softening. According to the previous studies [42], the stress-strain relationships of soils had six types. Consequently, the stress-strain relationships of samples in this study could be divided into strong softening type, general softening type, and weak softening type, respectively, which depended on the dry ranges of samples. Specifically, the stress-strain relationships of samples with dry range II and dry range III were general softening type and weak softening type, respectively, while the stress-strain relationships of samples with dry range I was strong softening type, indicating that the samples maintained high strength within a small strain. Additionally, compared with the stress-strain relationships under WD cycles, the stress-strain relationships of samples subjected to WDFT cycles entered the softening stage earlier, which indicated that the freeze-thaw process in the WDFT cycles promoted the softening of the stress-strain relationships.

4.2.2. Failure Strength. As previously described, all the stress-strain relationships of samples showed strain softening. Consequently, the failure strength of samples was estimated according to the peak values shown in Figure 12. Figure 13 shows the curves of failure strength variation with cycles. It is found that under the WD and WDFT cycles, the failure strength of samples with the same dry range has a similar variation trend, that is, decreased at the beginning of cycles and then tended to be stable. Among them, the first cycle resulted in a significant decrease of soil strength, which was consistent with that reported in the literature. After seven WD cycles and WDFT cycles, the failure strength of samples with dry range I decreased by $37.2 \%$ and $46.9 \%$, while the failure strength of samples with dry range II and dry range III decreased by $53.2 \%, 55.7 \%$ and $48.2 \%, 59.1 \%$, respectively. In addition, compared with the failure strength of samples subjected to WD cycles, the failure strength attenuation of samples subjected to WDFT cycles was more significant. It indicated that the freeze-thaw process in the WDFT cycles aggravated the failure strength attenuation of samples.

According to the previous studies [23-25], it is found that the average dry range of shallow foundation soil below the canals in the field was close to dry range II. For the purpose of accurately predicting the variation trend of the failure strength of foundation soils below the canal with WDFT cycles, the failure strength of samples (dry range II) with WDFT cycles shown in Figure 13(b) was fitted with a COD $\left(R^{2}\right)$ of 0.99 . The formula was as follows:

$$
f_{\mathrm{WDFT}}=27.1+30.9 \cdot e^{-1.44 \cdot N_{\mathrm{WDFT}}} \text {. }
$$

\section{Conclusions}

On the basis of the above studies, the following conclusions are drawn:

(1) During the WD cycles, the volume changes of expansive mudstone with a higher dry range would be more significant. The freeze-thaw process in the WDFT cycles resulted in a decrease of volume change ranges when the expansive mudstone had a relatively smaller dry range and a slightly increase of volume change ranges when the expansive mudstone had a relatively larger dry range.

(2) Under the cycles of WD or WDFT, the stress-strain relationships of samples with different dry ranges all presented strain softening. In the meantime, the freeze-thaw process in the WDFT cycles promoted the softening of the stress-strain relationships.

(3) During the WD/WDFT cycles, the first cycle resulted in a significant decrease of failure strength. After seven WD/WDFT cycles, the failure strength of expansive mudstones with different dry ranges decreased by $37.2 \% \sim 59.1 \%$. Additionally, the freezethaw process in the WDFT cycles aggravated the failure strength attenuation of expansive mudstones.

(4) Further study is encouraged on the investigation of phase change materials used to treat expansive mudstone under freeze-thaw/wet-dry-freeze-thaw cycles

\section{Data Availability}

The data used to support the findings of this study are included within the article.

\section{Conflicts of Interest}

The authors declare that they have no conflicts of interest.

\section{Acknowledgments}

This work was funded by the National Natural Science Foundation of China ( 51879166, 51778287, and 51708291) and the Open Research Fund Program of State Key Laboratory of Permafrost Engineering of China (SKLFSE201909).

\section{References}

[1] J. Q. Chen and J. Xia, "Facing the challenge: barriers to sustainable water resources development in China," Hydrological Sciences Journal, vol. 44, no. 4, pp. 507-516, 1999.

[2] J. Xu, Q. Z. Wang, J. L. Ding, Y. F. Li, S. H. Wang, and Y. G. Yang, "Frost heave of irrigation canals in seasonal frozen 
regions," Advances in Civil Engineering, vol. 2019, Article ID 2367635, 14 pages, 2019.

[3] S. Liu, Y. Lu, L. Weng, and F. Bai, "Field study of treatment for expansive soil/rock channel slope with soilbags," Geotextiles and Geomembranes, vol. 43, no. 4, pp. 283-292, 2015.

[4] Y. Lu, S. Liu, L. Weng, L. Wang, Z. Li, and L. Xu, "Fractal analysis of cracking in a clayey soil under freeze-thaw cycles," Engineering Geology, vol. 208, pp. 93-99, 2016.

[5] P. F. He, Y. H. Mu, Z. H. Yang, W. Ma, J. H. Dong, and Y. T. Huang, "Freeze-thaw cycling impact on the shear behavior of frozen soil-concrete interface," Cold Regions Science and Technology, vol. 173, Article ID 103024, 2020.

[6] L. Tang, S. Cong, L. Geng, X. Ling, and F. Gan, "The effect of freeze-thaw cycling on the mechanical properties of expansive soils," Cold Regions Science and Technology, vol. 145, pp. 197-207, 2018.

[7] L. Tang, S. Cong, X. Ling, W. Xing, and Z. Nie, "A unified formulation of stress-strain relations considering microdamage for expansive soils exposed to freeze-thaw cycles," Cold Regions Science and Technology, vol. 153, pp. 164-171, 2018.

[8] L. Miao, S. Liu, and Y. Lai, "Research of soil-water characteristics and shear strength features of Nanyang expansive soil," Engineering Geology, vol. 65, no. 4, pp. 261-267, 2002.

[9] Y. Lu, S. Liu, E. Alonso, L. Wang, L. Xu, and Z. Li, "Volume changes and mechanical degradation of a compacted expansive soil under freeze-thaw cycles," Cold Regions Science and Technology, vol. 157, pp. 206-214, 2019.

[10] Y. Lu, S. H. Liu, Y. G. Zhang, Z. Li, and L. Xu, "Freeze-thaw performance of a cement-treated expansive soil," Cold Regions Science and Technology, vol. 170, Article ID 102926, 2020.

[11] J. Q. Wang, Q. Wang, S. Lin, Y. Han, and N. Wang, "Relationship between the shear strength and microscopic pore parameters of saline soil with different freeze-thaw cycles and salinities," Symmetry, vol. 12, no. 10, p. 1709, 2020.

[12] Y. Liu, Q. Wang, S. Liu et al., "Experimental investigation of the geotechnical properties and microstructure of lime-stabilized saline soils under freeze-thaw cycling," Cold Regions Science and Technology, vol. 161, pp. 32-42, 2019.

[13] S. Li, Y. Lai, W. Pei, S. Zhang, and H. Zhong, "Moisturetemperature changes and freeze-thaw hazards on a canal in seasonally frozen regions," Natural Hazards, vol. 72, no. 2, pp. 287-308, 2014.

[14] Z. Li, J. Chen, and M. Sugimoto, "Pulsed NMR measurements of unfrozen water content in partially frozen soil," Journal of Cold Regions Engineering, vol. 34, no. 3, Article ID 04020013, 2020.

[15] S. Li, M. Zhang, Y. Tian, W. Pei, and H. Zhong, "Experimental and numerical investigations on frost damage mechanism of a canal in cold regions," Cold Regions Science and Technology, vol. 116, pp. 1-11, 2015.

[16] B. Kong, F. Xia, B. Yu, T. Xia, and Z. Ding, "Pore size changes in marine soft soil under various freezing conditions," Journal of Marine Science and Engineering, vol. 8, no. 3, p. 170, 2020.

[17] S. Suzuki, "Dependence of unfrozen water content in unsaturated frozen clay soil on initial soil moisture content," Soil Science and Plant Nutrition, vol. 50, no. 4, pp. 603-606, 2004.

[18] S. Wang, P. Yang, and Z. Yang, "Characterization of freezethaw effects within clay by 3D X-ray Computed Tomography," Cold Regions Science and Technology, vol. 148, pp. 13-21, 2018.

[19] J. K. Torrance, T. Elliot, R. Martin, and R. J. Heck, "X-ray computed tomography of frozen soil," Cold Regions Science and Technology, vol. 53, no. 1, pp. 75-82, 2008.
[20] J. Liu, P. Yang, L. Li, and T. Zhang, "Characterizing the pore size distribution of a chloride silt soil during freeze-thaw processes via nuclear magnetic resonance relaxometry," Soil Science Society of America Journal, vol. 84, no. 5, pp. 15771591, 2020.

[21] K. Watanabe and T. Wake, "Measurement of unfrozen water content and relative permittivity of frozen unsaturated soil using NMR and TDR," Cold Regions Science and Technology, vol. 59, no. 1, pp. 34-41, 2009.

[22] A. Liernur, A. Schomburg, P. Turberg, C. Guenat, R.-C. Le Bayon, and P. Brunner, "Coupling X-ray computed tomography and freeze-coring for the analysis of fine-grained lowcohesive soils," Geoderma, vol. 308, pp. 171-186, 2017.

[23] R. Zhu, Y. H. Huang, C. Zhang, W. L. Guo, and H. Chen, "Laboratory and centrifugal model tests on failure mechanism of canal slopes under cyclic action of wetting-drying," European Journal of Environmental and Civil Engineering, Article ID 1773321, 2020.

[24] R. Zhu, Z.-Y. Cai, Y.-H. Huang, C. Zhang, W.-L. Guo, and $\mathrm{X}$. Zhu, "Centrifugal and field studies on water infiltration characteristics below canals under wetting-drying-freezing-thawing cycles," Journal of Central South University, 2021.

[25] S. Y. Li, Y. M. Lai, M. Y. Zhang, W. S. Pei, C. Zhang, and F. Yu, "Centrifuge and numerical modeling of the frost heave mechanism of a cold-region canal," Acta Geotechnica, vol. 14, no. 12 , pp. 1113-1128, 2018.

[26] C. W. W. Ng, L. T. Zhan, C. G. Bao, D. G. Fredlund, and B. W. Gong, "Performance of an unsaturated expansive soil slope subjected to artificial rainfall infiltration," Géotechnique, vol. 53, no. 2, pp. 143-157, 2003.

[27] L. Miao, S. L. Houston, Y. Cui, and J. Yuan, "Relationship between soil structure and mechanical behavior for an expansive unsaturated clay," Canadian Geotechnical Journal, vol. 44, no. 2, pp. 126-137, 2007.

[28] C. Zhang, Z. Y. Cai, Y. H. Huang, and H. Chen, "Laboratory and centrifugal model tests on influence of swelling rock with drying-wetting cycles on stability of canal slope," Advances in Civil Engineering, vol. 2018, Article ID 4785960, 10 pages, 2018.

[29] Z. Y. Cai, R. Zhu, Y. H. Huang, C. Zhang, W. L. Guo, and $\mathrm{H}$. Chen, "Influences of freeze-thaw process on the deterioration mode of expansive soil canal slope," Journal of $\mathrm{Hy}$ draulic Engineering, vol. 51, no. 8, pp. 915-923, 2020, in Chinese.

[30] Z. Y. Cai, R. Zhu, Y. H. Huang, C. Zhang, and W. L. Guo, "Centrifugal model tests on deterioration process of canal under cyclic action of coupling wetting-drying and freezethaw," Chinese Journal of Geotechnical Engineering, vol. 42, no. 10, pp. 1773-1782, 2020, in Chinese.

[31] Z. Zeng, L. Kong, M. Wang, and H. M. Sayem, "Assessment of engineering behaviour of an intensely weathered swelling mudstone under full range of seasonal variation and the relationships among measured parameters," $\mathrm{Ca}$ nadian Geotechnical Journal, vol. 55, no. 12, pp. 1837-1849, 2018.

[32] ASTM, Standard Test Methods for Liquid Limit, Plastic Limit, and Plasticity Index of Soils (D4318), ASTM, West Conshohocken, PA, USA, 2010.

[33] ASTM, Standard Test Methods for Laboratory Compaction Characteristics of Soil Using Standard Effort (D698), ASTM, West Conshohocken, PA, USA, 2012.

[34] ASTM, Standard Test Method for Expansion Index of Soils (D4829), ASTM, West Conshohocken, PA, USA, 2019. 
[35] ASTM, Standard Test Methods for Particle-Size Distribution (Gradation) of Soils Using Sieve Analysis (D6913), ASTM, West Conshohocken, PA, USA, 2009.

[36] ASTM, Standard Test Method for Particle-Size Analysis of Soils (D422), ASTM, West Conshohocken, PA, USA, 2007.

[37] L. Xu, S. H. Liu, Y. Lu, Y. J. Song, and Q. Yang, "Physicomechanical properties of expansive soil under freeze-thaw cycles," Rock and Soil Mechanics, vol. 37, no. 2, pp. 167-174, 2016.

[38] A. R. Estabragh, B. Parsaei, and A. A. Javadi, "Laboratory investigation of the effect of cyclic wetting and drying on the behaviour of an expansive soil," Soils and Foundations, vol. 55, no. 2, pp. 304-314, 2015.

[39] J. Lu, M. Zhang, X. Zhang, W. Pei, and J. Bi, "Experimental study on the freezing-thawing deformation of a silty clay," Cold Regions Science and Technology, vol. 151, no. 5, pp. 19-27, 2018.

[40] K. Mu, L. W. Kong, X. W. Zhang, and S. Yin, "Experimental investigation on engineering behaviors of red clay under effect of wetting-drying cycles," Rock and Soil Mechanics, vol. 37, no. 8, pp. 2247-2253, 2016, in Chinese.

[41] J. Qi, W. Ma, and C. Song, "Influence of freeze-thaw on engineering properties of a silty soil," Cold Regions Science and Technology, vol. 53, no. 3, pp. 397-404, 2008.

[42] X. Y. Wu, Q. G. Liang, F. J. Niu, and C. Q. Li, "Study on hardened and softened classification in shear test," Chinese Journal of Underground Space and Engineering, vol. 13, no. 6, pp. 1457-1466, 2017, in Chinese. 